\title{
MiR-125b promotes cell migration and invasion by targeting PPP1CA-Rb signal pathways in gastric cancer, resulting in a poor prognosis
}

\author{
Jian-Guo Wu $\cdot$ Jin-Jie Wang $\cdot$ Xin Jiang $\cdot$ Jian-Ping Lan $\cdot$ \\ Xu-Jun He $\cdot$ Hui-Ju Wang $\cdot$ Ying-Yu Ma $\cdot$ Ying-Jie Xia $\cdot$ \\ Guo-Qing Ru $\cdot$ Jie Ma $\cdot$ Zhong-Sheng Zhao $\cdot$ Ren Zhou
}

Received: 20 February 2014/Accepted: 23 August 2014/Published online: 21 September 2014

(c) The International Gastric Cancer Association and The Japanese Gastric Cancer Association 2014

\begin{abstract}
Background MiR-125b functions as an oncogene in many cancers; however, its clinical significance and molecular mechanism in gastric cancers have never been sufficiently investigated. Here, we elucidated the functions and molecular regulated pathways of MiR-125b in gastric cancer.

Methods We investigated MiR-125b expression in fresh tissues from 50 gastric cancer patients and 6 gastric cancer cell lines using RT-PCR, and explored its prognostic value by hybridizing MiR-125b in situ for 300 clinical gastric tumor tissues with pathological diagnosis and clinical parameters. The effects of MiR-125b on gastric cancer cells and downstream target genes and proteins were analyzed by MTT, transwell assay, RT-PCR, and western blot on the basis of silencing MiR-125b in vitro. Luciferase reporter plasmid was constructed to demonstrate MiR125b's direct target.

Results MiR-125b was upregulated in gastric cancer tissues and cell lines, and significantly promoted cellular proliferation, migration, and invasion by downregulating the expression of PPP1CA and upregulating $\mathrm{Rb}$
\end{abstract}

J.-G. Wu $\cdot$ J.-J. Wang $\cdot$ X. Jiang $\cdot$ R. Zhou $(\bowtie)$

Department of Pathology and Pathophysiology, Zhejiang

University School of Medicine, Hangzhou 310058,

People's Republic of China

e-mail: zhouren@zju.edu.cn

J.-P. Lan · G.-Q. Ru · J. Ma · Z.-S. Zhao ( $₫)$

Department of Pathology, Zhejiang Provincal People's Hospital,

Hangzhou 310014, People's Republic of China

e-mail: zhaozhongsheng1950@163.com

X.-J. He · H.-J. Wang · Y.-Y. Ma · Y.-J. Xia

Key Laboratory of Gastroenterology of Zhejiang Province,

Hangzhou 310014, People's Republic of China phosphorylation. MiR-125b expression was significantly correlated with tumor size and depth of invasion, lymph nodes, distant metastasis, and TNM stage. The high-MiR$125 \mathrm{~b}$-expression group had a significantly poorer prognosis than the low-expression group $(P<0.05)$ in stages I, II, and III, and the 5-year survival rate in of the highexpression group was significantly lower than that of the low-expression group.

Conclusions MiR-125b functions as an oncogene by targeting downregulated PPP1CA and upregulated $\mathrm{Rb}$ phosphorylation in gastric cancer. MiR-125b not only promotes cellular proliferation, migration, and invasion in vitro, but also acts as an independent prognostic factor in gastric cancer.

Keywords MiR-125b - PPP1CA · Gastric cancer

\section{Introduction}

Gastric cancer is the most frequent cause of cancer-related death, second only to lung cancer. Its mortality rate is the highest among all cancers in China, and it represents $25 \%$ of gastric cancer mortality worldwide. The overall 5-year survival rate in China is poor at $40 \%$ because most gastric cancers are diagnosed at stage III or IV and have a high rate of lymph node metastasis (50-75\%) [1]; therefore, it is of great clinical value to further elucidate the molecular mechanisms in gastric cancer and develop reliable earlier diagnostic markers and new therapeutic strategies.

MicroRNAs are a family of 21- to 25-nucleotide, noncoding small RNAs that primarily function as gene regulators by regulating messenger RNA (mRNA) translation and degradation [2]. MicroRNAs play a role in cancer or cancer prevention by modulating the expression of 
downstream mRNAs [3]. MiR-125b acts as a tumor suppressor or oncogene such as downregulated in ovarian carcinoma and thyroid carcinoma [4-7] but elevated in pancreatic cancer, oligodendroglial tumors, prostate cancer, myelodysplastic syndrome, and acute myeloid leukemia [4, 8-10]; however, nothing is known about the expression and biological role of MiR-125b in gastric cancer. In this study, we evaluated the expression of MiR$125 \mathrm{~b}$ and attempted to clarify its clinical prognostic value in gastric cancer. Furthermore, the effect of MiR-125b expression on gastric cancer cell proliferation, migration, and invasion was analyzed in vitro using a microRNA silencing technique. We also demonstrated the direct target of MiR-125b by luciferase reporter assay, RT-PCR, and western blot.

\section{Materials and methods}

\section{Reagents}

Four synthetic, chemically modified, short single- or double-stranded RNA oligonucleotides, MiR-125b mimic (MiR-125bm), MiR-125b mimic negative control (MiR125bm NC), MiR-125b inhibitor (MiR-125bi), and MiR125b inhibitor NC (MiR-125bi NC), were synthesized from Exiqon Co., Ltd. (Copenhagen, Denmark).

Frozen fresh tissues, tissue microarrays, and cell cultures

Fresh gastric cancer tissues and adjacent non-tumor tissues were obtained from 50 patients with primary gastric cancer at the Zhejiang Provincial People's Hospital, Hangzhou, China. All patients provided informed consent before surgery for the use of their tissues. After surgical removal, tissues were immediately frozen in liquid nitrogen and stored at $-80{ }^{\circ} \mathrm{C}$ until used. A tumor tissue microarray of paraffinized specimens from 300 gastric cancer patients who underwent curative gastrectomy between 2003 and 2008 at the Department of Surgery (Zhejiang Provincial People's Hospital, Hangzhou, China) was included. Written informed consent for experimental immunohistochemistry was obtained from all patients before analysis. The gastric cancer patients ranged in age from 17 to 80 years (median, 66.0 years) and had received no radiotherapy or chemotherapy before surgery. All cases were classified according to the World Health Organization's pathological classification of tumors. The clinicopathological characteristics of the gastric cancer patients are summarized in Table 1. Full follow-up data for 5 years until 2012 were recorded for all patients.

Human gastric cancer cell lines BGC-823, SGC-7901, AGS, MKN-45, MKN-28, and HGC-27 and non-malignant
Table 1 Relationship between miR-125b expression and clinicopathological factors

\begin{tabular}{|c|c|c|c|c|}
\hline \multirow{2}{*}{$\begin{array}{l}\text { Clinical } \\
\text { parameter }\end{array}$} & \multicolumn{4}{|l|}{$\operatorname{miR}-125 b$} \\
\hline & Low & High & $\mathrm{t} / \mathrm{X} 2$ & $P$ \\
\hline $\begin{array}{l}\text { Age (years), } \\
\text { mean } \pm S D\end{array}$ & $65.27 \pm 9.28$ & $67.16 \pm 10.02$ & -1.658 & 0.098 \\
\hline Sex & & & 1.981 & 0.159 \\
\hline Male & $84(39.4 \%)$ & $129(60.6 \%)$ & & \\
\hline Female & $42(48.3 \%)$ & $45(51.7 \%)$ & & \\
\hline Location & & & 3.376 & 0.185 \\
\hline Distal & $75(46.9 \%)$ & $85(53.1 \%)$ & & \\
\hline Middle & $29(35.8 \%)$ & $52(64.2 \%)$ & & \\
\hline Proxima & $22(37.3 \%)$ & $37(62.7 \%)$ & & \\
\hline Size & & & 68.1 & 0.001 \\
\hline$<5 \mathrm{~cm}$ & $90(68.7 \%)$ & $41(31.32 \%)$ & & \\
\hline$>5 \mathrm{~cm}$ & $36(21.3 \%)$ & $133(78.7 \%)$ & & \\
\hline Histological grade & & & 9.849 & 0.02 \\
\hline Well & $12(70.6 \%)$ & $5(29.4 \%)$ & & \\
\hline Moderately & $42(47.7 \%)$ & $46(52.3 \%)$ & & \\
\hline Poorly & $66(36.1 \%)$ & $117(63.9 \%)$ & & \\
\hline Others & $6(50.0 \%)$ & $6(50.0 \%)$ & & \\
\hline Invation depth & & & 27.58 & 0.001 \\
\hline $\mathrm{T} 1$ & $13(86.7 \%)$ & $2(13.3 \%)$ & & \\
\hline $\mathrm{T} 2$ & $19(73.1 \%)$ & $7(26.9 \%)$ & & \\
\hline $\mathrm{T} 3$ & $75(38.3 \%)$ & $121(61.7 \%)$ & & \\
\hline $\mathrm{T} 4$ & $19(29.7 \%)$ & $45(70.3 \%)$ & & \\
\hline TNM stages & & & 94.58 & 0.001 \\
\hline I & $16(72.7 \%)$ & $6(27.3 \%)$ & & \\
\hline II & $75(76.5 \%)$ & $23(23.5 \%)$ & & \\
\hline III & $27(18.1 \%)$ & $122(81.9 \%)$ & & \\
\hline IV & $8(25.0 \%)$ & $24(75.0 \%)$ & & \\
\hline $\begin{array}{l}\text { Lymphatic } \\
\text { metastasis }\end{array}$ & & & 61.23 & 0.001 \\
\hline No & $56(83.6 \%)$ & $11(16.4 \%)$ & & \\
\hline Yes & $70(30.0 \%)$ & $163(70.0 \%)$ & & \\
\hline $\begin{array}{l}\text { Regional lymph } \\
\text { nodes }\end{array}$ & & & 84.16 & 0.001 \\
\hline PNO & $56(83.6 \%)$ & $11(16.4 \%)$ & & \\
\hline PN1 & $28(54.9 \%)$ & $23(45.1 \%)$ & & \\
\hline $\mathrm{PN} 2$ & $28(32.9 \%)$ & $57(67.1 \%)$ & & \\
\hline PN3 & $14(14.4 \%)$ & $83(85.6 \%)$ & & \\
\hline Distant metastasis & & & 4.25 & 0.039 \\
\hline No & $118(44.0 \%)$ & $150(56.0 \%)$ & & \\
\hline Yes & $8(25.0 \%)$ & $24(75.0 \%)$ & & \\
\hline
\end{tabular}

gastric epithelial cell line GES-1 were obtained from Shanghai Institute of Digestive Surgery (Shanghai, China) and cultured in RPMI1640 containing $10 \%$ fetal bovine serum (FBS), $50 \mathrm{U} / \mathrm{mL}$ penicillin, and $50 \mu \mathrm{g} / \mathrm{mL}$ streptomycin. All cells were maintained at $37^{\circ} \mathrm{C}$ under an atmosphere of $5 \% \mathrm{CO}_{2}$. 
In situ detection of MiR-125b

In situ hybridization was performed using the sensitivity enhanced in situ hybridization kit (MK1030) (Boster Biological Technology Co., Ltd., Wuhan, China) according to the manufacturer's instructions. Digoxin-labeled MiR-125b probe (MiRCURY LNA Detection Probe, 250 pmol, 3'DIG labeled, Exiqon, Copenhagen, Denmark) was used to detect cytoplasmic MiR-125b. The degree of immunostaining was reviewed and scored independently by 2 observers based on the proportion of positively stained tumor cells and stain intensity [11, 12]. Tumor cell proportion was scored as follows: $0(\leq 5 \%$ positive tumor cells), 1 (6-25\% positive tumor cells), 2 (26-50\% positive tumor cells), and 3 ( $>51 \%$ positive tumor cells). Staining intensity was graded according to the following criteria: 0 (no staining), 1 (weak staining, light yellow), 2 (moderate staining, yellow brown), and 3 (strong staining, brown). The staining index was calculated as the product of staining intensity score and the proportion of positive tumor cells. Using this method of assessment, we evaluated MiR-125b expression in benign gastric epithelia and malignant lesions by determining the staining index with scores of $0,1,2,3,4,6$, or 9 . The cutoff value for high and low expression levels was chosen based on a measure of heterogeneity using the log-rank test with respect to overall survival. The optimal cutoff value was identified as a staining index score of 4 or higher to define tumors with high MiR-125b expression, and a staining index score of 3 or lower to define those with low MiR-125b expression.

\section{MiR-125b transfection}

MiR-125bi, MiR-125bi NC, MiR-125bm, and MiR-125bm NC were transfected into HGC-27, MKN-28, and MKN-45 cells, respectively, grown in 6-well dishes $\left(2.0 \times 10^{5}\right.$ cells per well) $24 \mathrm{~h}$ before transfection. Transfection was performed with Lipofectamine 2000 (Invitrogen) according to the reagent protocol. Twenty-four hours after transfection, the cells were harvested and used for proliferation, migration, invasion, and clone-formation assays.

\section{SiRNA targeting}

To inhibit expression of endogenous PPP1CA, high-performance liquid chromatography-purified PPP1CA small interfering (si) RNA (sense: 5'-AGUACUACGACCUU CUGCGTT- $3^{\prime}$ ), and a scrambled siRNA with no homology to any known human mRNA and used as an NC (sense: $5^{\prime}$ GUUCUCCGAACGUGUCACGTT- $3^{\prime}$ ) were designed and prepared according to the sequence of the PPP1CA gene. SiRNA oligonucleotide duplexes were synthesized and chemically modified (Invitrogen).
RT-qPCR quantification of MiR-125b and its target gene

RT-qPCR was performed to determine the expression of mature MiR-125b. Briefly, total RNA was extracted from tissues and cells using TRIzol (Invitrogen) according to the manufacturer's instructions. Complementary (c) DNA synthesis was carried out with the superscript III cDNA synthesis kit (Invitrogen) using $1 \mu \mathrm{g}$ total RNA as the template and specific reverse primers under $16{ }^{\circ} \mathrm{C}$ for $30 \mathrm{~min}, 42{ }^{\circ} \mathrm{C}$ for $30 \mathrm{~min}$, and $85^{\circ} \mathrm{C}$ for $5 \mathrm{~min}$ of reverse transcription. The resulting cDNA was amplified by PCR using MiR-125b and U6 (endogenous control) specific primers with SYBR Premix Ex Taq (Takara). Specific primers for MiR-125b were 5'-TCCCTGAGACCCTAA CTTGTG-3'; specific primers for U6 were $5^{\prime}$-ACG CAAATTCGTGAAGCGTT- $3^{\prime}$. PCR parameters were as follows: $95{ }^{\circ} \mathrm{C}$ for $5 \mathrm{~min}$, followed by 40 cycles at $95{ }^{\circ} \mathrm{C}$ for $10 \mathrm{~s}, 60{ }^{\circ} \mathrm{C}$ for $20 \mathrm{~s}$, and $72{ }^{\circ} \mathrm{C}$ for $20 \mathrm{~s}$. At the end of the PCR cycles, a melting-curve analysis was performed. The expression of MiR-125b in tissues was compared to 1 non-tumor sample using the $2^{-\Delta \Delta C T}$ method, and the expression of MiR-125b in gastric cancer cells was compared to GES-1.

RT-qPCR was performed to determine the effect of MiR-125b on its target gene PPP1CA in gastric cancer cells. Briefly, total RNA was extracted from MKN-28 and HGC-27 cell lines transfected with MiR-125b inhibitor or an NC, and used as a template to synthesize cDNA. The resulting cDNA was amplified by PCR using PPP1CAgene-specific primers (primers sense: 5'-TATTTG AGTATGGCGGTTTCC-3' ${ }^{\prime}$, antisense: $5^{\prime}$-CCACAGTTTG ATGTTGTAGCG- $3^{\prime}$ ) and GAPDH (endogenous control) gene specific primers (primers sense: $5^{\prime}$-TGAAGGTCG GAGTCAACGG-3', antisense: 5'-CTGGAAGATGGTG ATGGGATT-3') with SYBR Premix Ex Taq (Takara).

Cell proliferation, migration, invasion, and clone-formation assays in vitro

Twenty-four hours or $72 \mathrm{~h}$ after transfection, MKN-28, HGC-27, and MKN-45 cells were harvested and subjected to the following assays. For the proliferation assay evaluated by the MTT test, cells were plated into 96-well plates in $1 \times 10^{3}$ cells/well in quintuplicate and allowed to adhere overnight. Ten microliters MTT $(5 \mathrm{mg} / \mathrm{mL})$ (SigmaAldrich Co., LLC, St. Louis, MO, USA) were added to each well $72 \mathrm{~h}$ later, and the cells were incubated for an additional $4 \mathrm{~h}$. Media was then removed and $150 \mu \mathrm{l}$ DMSO was added. Absorbance $(A)$ at $570 \mathrm{~nm}$ and $630 \mathrm{~nm}$ was measured using a microplate reader. The relative cell proliferation (\%) is shown by the following equation: relative proliferation rate $(\%)=\left(\mathrm{A}_{570 \mathrm{~nm}}-\mathrm{A}_{630 \mathrm{~nm}}\right)$ of study group/ 
$\left(A_{570 n m}-A_{630 n m}\right)$ of control group $\times 100 \%$. For migration assays, transfected cells $\left(6 \times 10^{4}\right.$ per well) were plated in the top chamber of a Transwell system (EMD Millipore Corp., Billerica, MA, USA), comprising a membrane with 8 - $\mu \mathrm{m}$-diameter pores in $200 \mu \mathrm{L}$ serum-free RPMI- 1640 . The inserts were placed into the bottom chamber wells of a 24-well plate containing RPMI-1640 with $20 \%$ FBS as a chemoattractant. After $24 \mathrm{~h}$ of incubation, cells remaining on the top layer were removed by scrubbing with a cotton swab, and cells on the lower surface of the membrane were fixed in $95 \%$ methanol for $15 \mathrm{~min}$, followed by staining with hematoxylin and eosin (HE).

For invasion assays, transfected cells $\left(1.5 \times 10^{5}\right.$ per well $)$ were plated into the top chamber with a matrigel-coated membrane, while the bottom chamber was filled with conditioned medium. After incubation for $48 \mathrm{~h}$, migrated cells (lower side of the membrane) were stained as described above. For clone-formation assays, after transfection for $24 \mathrm{~h}$, cells were harvested and adjusted to a low density (200 cells/well of a 6-well plate) and incubated for 2 or 3 weeks at $37{ }^{\circ} \mathrm{C}$ in a humidified atmosphere containing $5 \% \mathrm{CO}_{2}$, with the culture medium changed every 3 days. Colony formation and growth were visualized using HE staining.

\section{Construction of reporter plasmids and luciferase assay}

To construct a reporter plasmid containing the $3^{\prime} \mathrm{UTR}$ of PPP1CA, the wild and mutated $3^{\prime}$-UTRs of the PPP1CA gene (wild sense: $5^{\prime}$-TCTAGAATCCTGGGGCCGAGGCT GCAGCTCAGGGCAA-3'; antisense: 5'-AGATCTTTGCC CTGAGCTGCAGCCTCGGCCCCAGGAT- $3^{\prime}$ and mutated sense: $5^{\prime}$-TCTAGAATCCTGGGGCTGGAGCTGCAGCCT GAAACAA-3'; antisense: 5'-AGATCTTTGTTTCAGGC TGCAGCTCCAGCCCCAGGAT- ${ }^{\prime}$ ) were synthesized and cloned into the XbaI site downstream of PGL3 and named pGL3-PPP1CA-3'UTR-WT and pGL3-PPP1CA-3'UTR-MT, respectively. MKN-28 cells $\left(1 \times 10^{5}\right)$ were seeded into 24-well plates and each was transfected with $0.5 \mu \mathrm{g}$ of either pGL3-PPP1CA-3'UTR-WT vector or pGL3-PPP1CA$3^{\prime}$ UTR-MT vector containing firefly luciferase, together with $0.2 \mu \mathrm{g}$ of the pRL-TK vector (Promega) containing Renilla

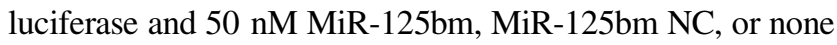
using the Lipofectamine 2000 (Invitrogen), transfection reagent according to the manufacturer's protocol. After transfection for $48 \mathrm{~h}$, luciferase activity was measured using the dual luciferase reporter assay system (Promega). The results were expressed as relative luciferase activity (firefly LUC/Renilla LUC).

Western blot analysis

Total protein extracts from each group of cells were resolved by $10 \%$ SDS-PAGE and transferred onto polyvinylidene fluoride (PVDF) (Millipore) membranes. After blocking, the PVDF membranes were washed 4 times for 15 min with tris-buffered saline with Tween 20 (TBST) at room temperature and incubated with primary antibody (rabbit anti-human PPP1CA, Rb, and Rb phospho monoclonal antibody; 1:1000, Epitomics, Inc., Burlingame, CA, USA). Following extensive washing, membranes were incubated with secondary peroxidase-linked goat anti-rabbit $\operatorname{IgG}(1: 1000$, Epitomics, Inc) for $1 \mathrm{~h}$. After washing again 4 times for $15 \mathrm{~min}$ with TBST at room temperature, the immunoreactivity was visualized by enhanced chemiluminescence (ECL kit, Pierce Biotechnology, Rockford, IL, USA), and membranes were exposed to Kodak XAR-5 films (Sigma-Aldrich).

Statistical analysis

All statistical analyses were conducted using Graphpad Prism 6 . The value of $P<0.05$ was considered statistically significant and error bars represent standard error of the mean (SEM). All experiments were repeated 3 times and a representative experiment result was shown with SEM.

\section{Results}

MiR-125b is upregulated in gastric tumor tissues and cell lines

The expression of MiR-125b in 50 pairs of matched gastric tumor tissues was analyzed using RT-qPCR. The expression levels of MiR-125b were much higher in gastric tumors $(0.37 \pm 0.23)$ than in non-tumor tissues $(0.19 \pm 0.13 ; P<0.01 ;$ Fig. 1a), while in the gastric tumor group, the lymphatic metastasis group $(n=32)$ $(0.47 \pm 0.23)$ was higher than non-lymphatic metastasis group $(n=18)(0.25 \pm 0.12 ; P<0.05$; Fig. 1a). In addition, we examined the MiR-125b expression of gastric cancer cell lines HGC-27, MKN-28, BGC-823, SGC-7901, AGS, and MKN-45 and the non-malignant gastric epithelial cell line GES-1. As shown in Fig. 1b, except for MKN45, gastric cancer cell lines expressed higher levels of MiR-125b than GES-1.

MiR-125b promoted gastric cancer cell proliferation, clone formation, migration, and invasion in vitro

To study the biological role of MiR-125b in gastric cancer, MiR-125bi, NC, and MiR-125bm NC were transfected into HGC-27, MKN-28, and MKN-45 cells, respectively. The effect of MiR-125b on the proliferation of human gastric cancer cell lines was evaluated using MTT assay, and the proliferation and clone formation of gastric cancer cells 

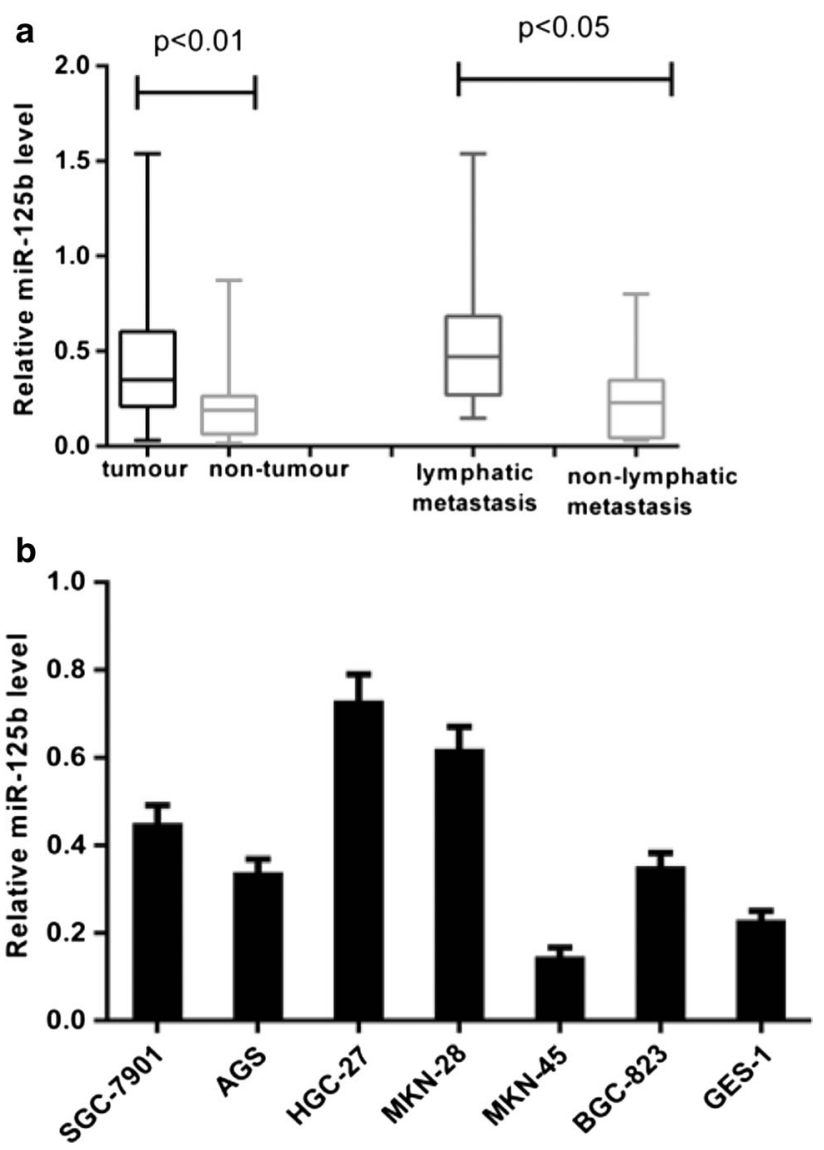

Fig. 1 Up-regulated expression of miR-125b in gastric tumour tissues and cell lines. a Relative expression of miR-125b in the gastric tumours tissues compared to corresponding non-tumour tissues. b Relative expression of miR-125b in gastric cancer cell lines compared to GES-1

transfected with MiR-125bi or MiR-125bm were measured. MiR-125bi significantly inhibited the proliferation and clone formation of HGC-27 and MKN-28 cells, and the relative proliferation and cell clone formation inhibition rates were 58 and $62 \%$ (Fig. $2 \mathrm{a}, P<0.01$ ), and 48 and $49 \%$ (Fig. 2b, c; $P<0.01$ ), respectively. In contrast, MiR$125 \mathrm{bm}$ significantly promoted the proliferation and clone formation of MKN-45 cells, and the relative proliferation and cell clone formation promotion rates were 132 and $138 \%$ (Fig. 2a, b; $P<0.01$ ), respectively. We further analyzed the effects of MiR-125b on the migratory and invasive behavior of gastric cell lines. As shown in Fig. 3, gastric cancer cells transfected with MiR-125bi displayed significantly lower transmembrane migration and invasion capacity (Fig. 3a-d) but those transfected with MiR-125bm displayed higher transmembrane migration capacity compared with those transfected with NC (Fig. 3e, f). The

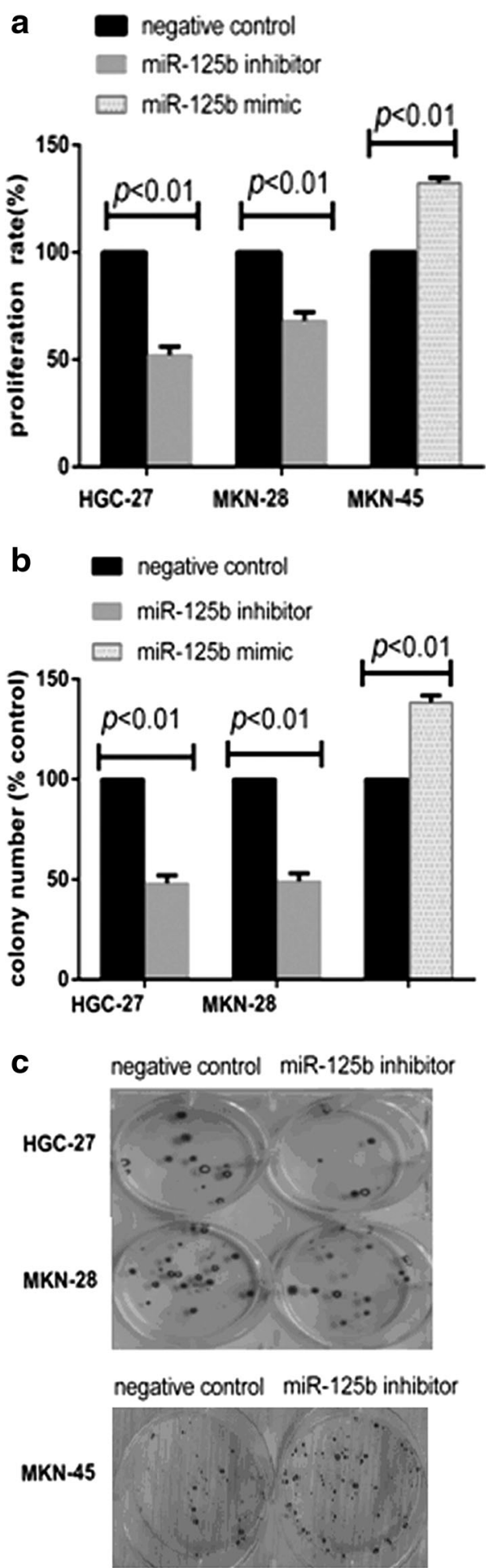

Fig. 2 MiR-125b significantly promoted proliferation and clone formation of HGC-27, MKN-28 and MKN-45 cells. a Relative proliferation rate of miR-125b negative control compared to miR125b inhibitor and miR-125b mimic $(P<0.01)$. b, c Relative clone formation rate miR-125b negative control compared to miR-125b inhibitor and miR-125b mimic $(P<0.01)$ 
Fig. 3 MiR-125b significantly promoted migration and invasion of HGC-27, MKN-28 and MKN-45 cells. a-d Relative migration and invasion inhibited rate of miR-125b negative control compared to miR-125b inhibitor $(P<0.01)$ and representative fields of migrated or invasive cells on membrane in HGC-27 and MKN-28 cell lines. e, f Relative migration and invasion promoted rate of $\mathrm{miR}$ $125 \mathrm{~b}$ negative control compared to miR-125b mimic $(P<0.01)$ and representative fields of migrated or invasive cells on membrane in MKN-45 cell line
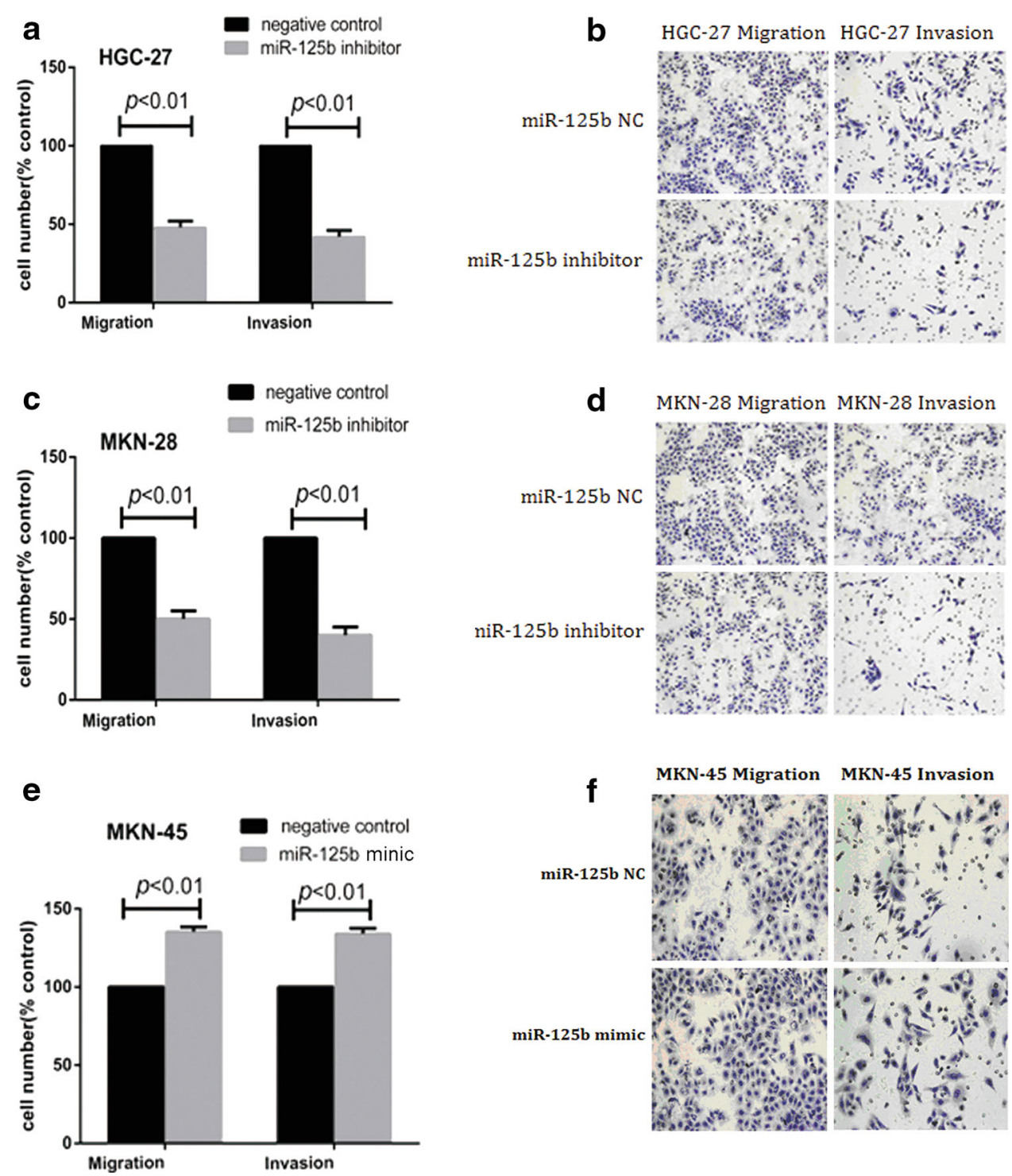

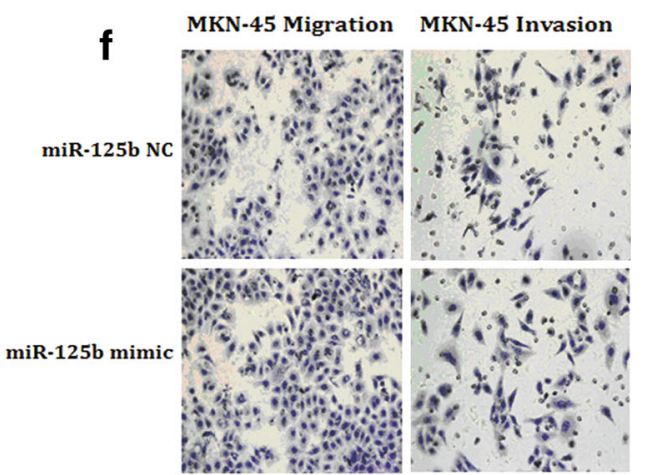

migration activity of MiR-125bi-transfected HGC-27 and MKN-28 was specifically reduced by approximately 48 and $50 \%$, respectively (Fig. 3a, c). The invasion activity was substantially reduced by approximately 42 and $40 \%$, respectively (Fig. 3a, c); however, the migration and invasion activity of MiR-125bm-transfected MKN-45 were specifically increased by approximately 135 and $134 \%$, respectively (Fig. 3e). These findings suggest that the MiR$125 \mathrm{~b}$ level is closely associated with the invasion and migration of gastric cancer cell lines.

MiR-125b directly repressed the expression of PPP1CA and promoted $\mathrm{Rb}$ phosphorylation in gastric cancer cells

TargetScan, Pictar-Vert, and Microrna Org were used to explore the potential targets of MiR-125b that might influence proliferation and the migration ability of cells. PPP1CA was found to be among the potential targets of the MiR-125b combination predicted by the three programs. The $3^{\prime}$ UTR of PPP1CA carries a binding site for MiR-125b (Fig. 4a), suggesting that PPP1CA mRNA might be a direct target of MiR-125b. To verify that the putative MiR-125b binding site in the $3^{\prime} \mathrm{UTR}$ of PPP1CA was responsible for regulation by MiR-125b, vectors were constructed containing wild-type or mutant $3^{\prime}$ UTR of PPP1CA co-transfected into MKN-28 cells with MiR-125bm, MiR-125bm $\mathrm{NC}$, or none. The transfection efficiency was normalized by co-transfection with Renilla reporter vector. As shown in Fig. 4b, MiR-125b significantly decreased the relative luciferase activity of wild-type PPP1CA $3^{\prime}$ UTR ( $\left.\sim 60 \%\right)$, suggesting that MiR-125b could directly bind to the $3^{\prime} \mathrm{UTR}$ of PPP1CA. In addition, we analyzed the effect of MiR$125 \mathrm{~b}$ on the expression of PPP1CA and $\mathrm{pRb}$ of the PPP1CA 
a mir-125b $3^{\prime}$...AGUGUUCAAUCCCAGAGUCCCU... Wild type PPP1CA 3'-UTR 5'...GGCCGAGGCUGCAGCUCAGGGC... Mutant PPP1CA3'-UTR 5'...GGCUGGAGCUGCAGCCUGAAAC...

b
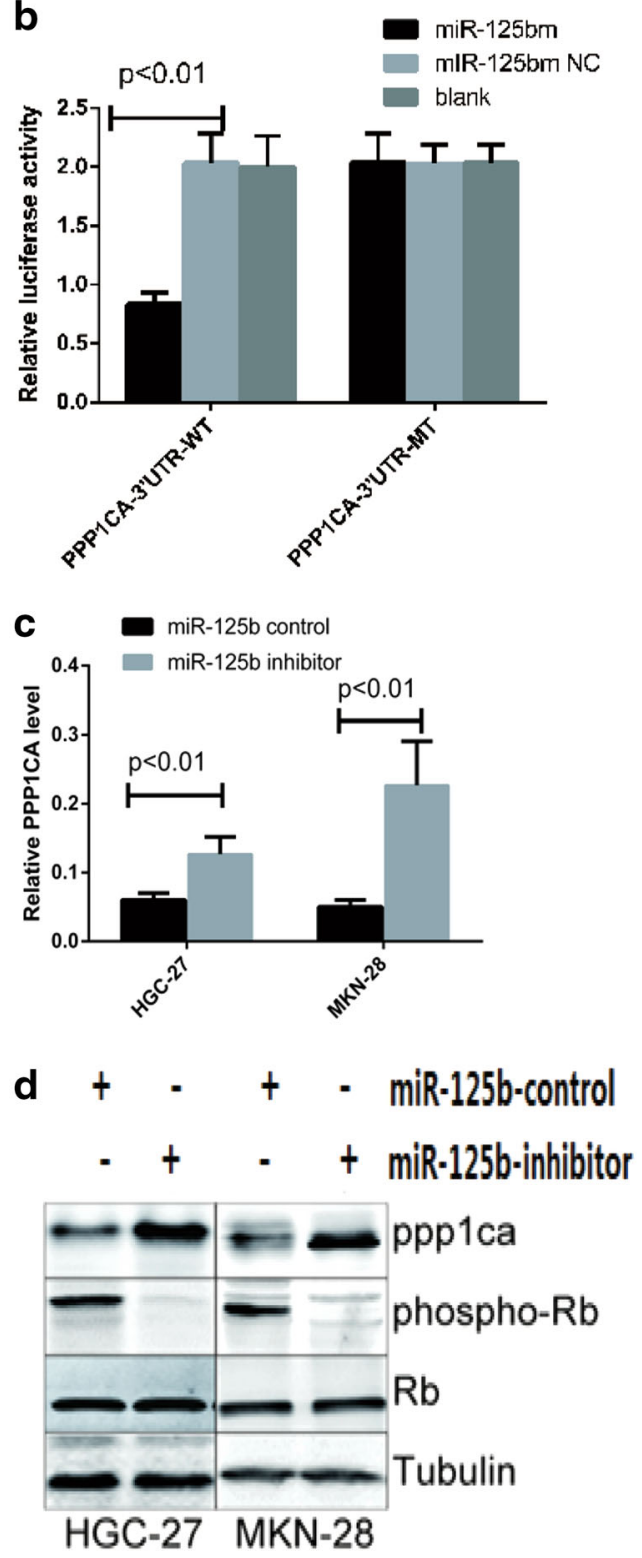

Fig. 4 MiR-125b directly repressed the expression of PPP1CA, and promoted $\mathrm{Rb}$ phosphorylation in gastric cancer cells. a Putative binding sites of miR-125b in the PPP1CA $3^{\prime}$-UTR predicted by Targetscan. b MiR-125bm significantly down-regulated luciferase activity controlled by wild-type PPP1CA $3^{\prime}$ UTR $(P<0.01)$, but did not affect luciferase activity controlled by mutant PPP1CA $3^{\prime} \mathrm{UTR}$. c MiR-125b inhibitor significantly promoted the expression of PPP1CA mRNA in HGC-27 and MKN-28 cell lines by RT-PCR. $(P<0.01)$. d MiR-125b inhibitor increased the expression of PPP1CA protein and decreased the expression $\mathrm{Rb}$ protein phosphorylation in HGC-27 and MKN-28 cell lines by western blot downstream target. RT-qPCR and/or western blot were performed to determine the mRNA and/or protein levels of these genes in HGC-27 and MKN-28 transfected MiR125bi or NC. As shown in Fig. 4c, d, the mRNA and protein levels of PPP1CA in MiR-125b-transfected HGC-27 and MKN-28 cells were much higher than in the control-transfected group, while the $\mathrm{Rb}$ protein phosphorylation level in MiR-125b-transfected HGC-27 and MKN-28 cells were much lower than in the control group (Fig. 4d). Taken together, these findings indicated that PPP1CA was a direct downstream target for MiR-125b in gastric cancer cells.

The inhibition of the proliferation, clone formation, invasion, and migration of HGC-27 and MKN-28 by MiR-125bi could be significantly attenuated by suppression of PPP1CA.

To address whether the above-observed phenotype is indeed caused by the suppression of PPP1CA from MiR-125b targeting other cellular genes, a rescue experiment was performed. MiR-125bi plus siPPP1CA (PPP1CA siRNA), MiR-125bi plus siPPP1CA NC, and siPPP1CA NC were transfected into HGC-27 and MKN-28 cells. After $48 \mathrm{~h}$ post-transfection, HGC-27 and MKN-28 co-transfected with MiR-125b inhibitor plus siPPP1CA revealed a higher effect than in those transfected with MiR-125bi plus siPPP1CA NC on enhancing cell proliferation, clone formation, migration, and invasion ability (Fig. 5a-d, $P<0.01$ ). Figure 5e showed the mRNA levels of PPP1CA in MiR125bi plus siPPP1CA co-transfected HGC-27 and MKN-28 cells were much lower than in the MiR-125bi plus siPPP1CA NC co-transfected groups (Fig. 5e, $P<0.01$ ). These results imply that suppressing PPP1CA expression could significantly attenuate the inhibitory effect of MiR$125 \mathrm{bi}$ on cell proliferation, clone formation, migration, and invasion, suggesting that the MiR-125b promoted the proliferation, clone formation, migration, and invasion migration of gastric cancer cells through targeting PPP1CA.

MiR-125b is an independent prognostic factor in gastric cancer

High levels of MiR-125b expression were detected in 174 (58\%) tumors, and low levels of MiR-125b expression were detected in 126 (42\%) tumors. MiR-125b was mainly localized in the cytoplasm of gastric cancer cells (Fig. 6a). Higher expression levels of MiR-125b correlated with the size of tumor, depth of invasion, lymph node, distant metastasis, and TNM stage $(P<0.05$, Table 1$)$, but was 
Fig. 5 Repressing PPP1CA could significantly attenuate the inhibitory effect of miR-125bi on HGC-27 and MKN-28 cells. a, b The proliferation and clone formation of HGC-27 and MKN-28cells co-transfected with miR-125bi plus siPPP1CA were significantly higher than those of cells transfected with miR-125bi plus siPPP1CA NC $(P<0.01)$. c, d The migration and invasion of HGC-27 and MKN-28cells co-transfected with miR-125bi plus siPPP1CA were significantly higher than those of cells transfected with miR-125bi plus siPPP1CA NC $(P<0.01)$. e The mRNA levels of PPP1CA in miR-125bi plus siPPP1CA co-transfected HGC27 and $\mathrm{MKN}-28$ cells were much lower than in the miR125bi plus siPPP1CA NC cotransfected groups $(P<0.01)$
SiPPP1CA NC

miR-125bi +siPPP1CA NC

a

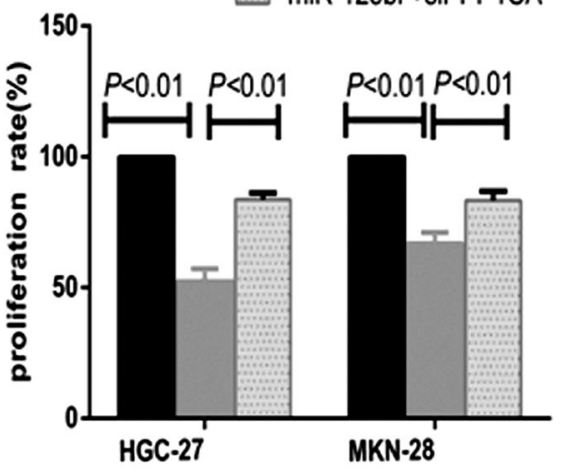

SIPPPCA NC

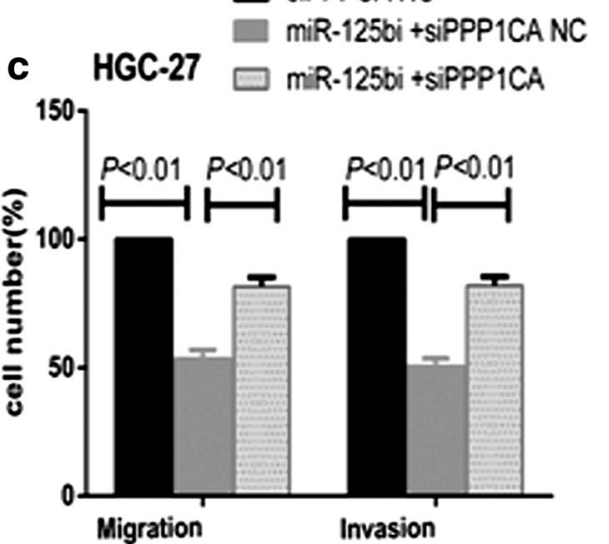

siPpP1CA NC

miR-125bi +siPPP1CA NC

e

miR-125bi + siPpP1CA

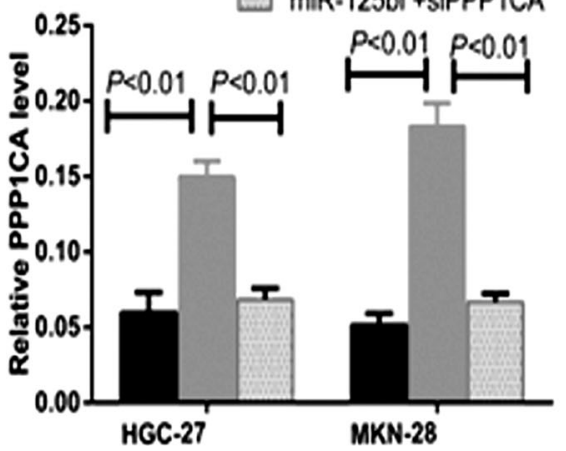

SiPPP1CA NC

miR-125bi +siPPP1CA NC

miR-125bi +siPPP1CA
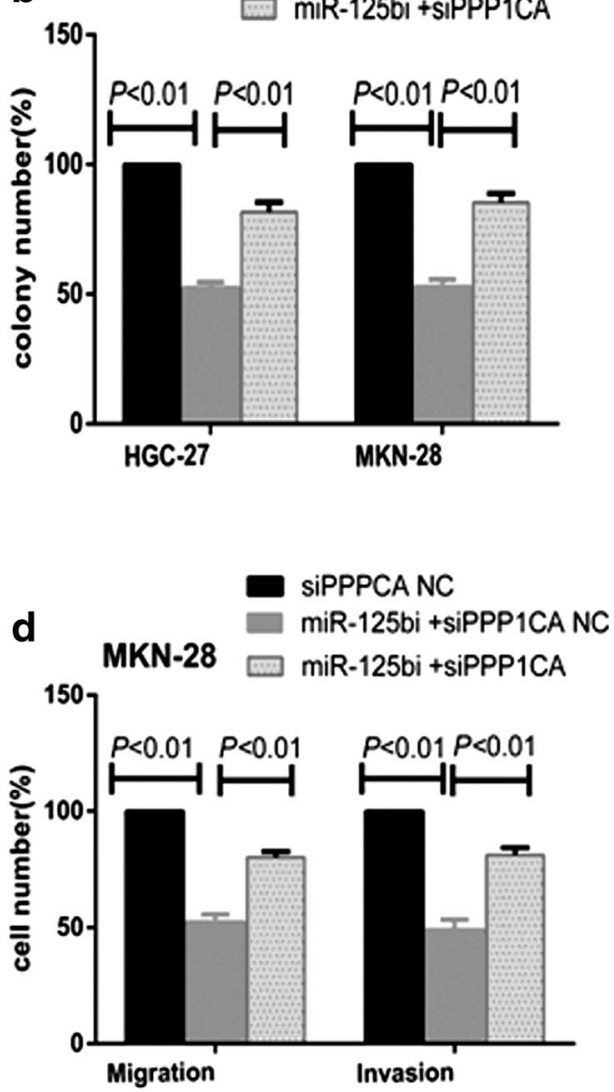

not associated with age, sex, location, and histological grade of gastric tumors $(P>0.05$, Table 1$)$.

We also analyzed the relationship between MiR-125b expression level and prognosis of gastric cancer (Fig. 6b). In stages I, II, and III, the 5-year survival rate of patients with gastric cancer and with high levels of MiR-125b expression was significantly lower than that in patients with low levels of expression $(P<0.05)$. But in stage IV, the expression of MiR-125b did not correlate with the 5 -year survival rate $(P=0.24)$. Multivariate cox regression analysis revealed that lymph node $(P<0.001)$, distant metastases $(P<0.001)$, TNM stage $(P=0.003)$, invasion depth $(P<0.001)$, and expression of MiR-125b $(P<0.001)$ were significantly different in gastric cancer survival; however, no significant differences were observed regarding size of tumor $(P=0.745)$, histological grade 
a i

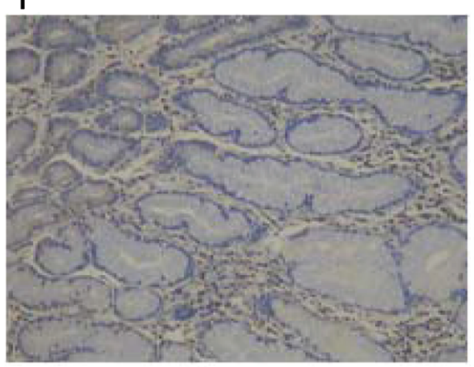

b
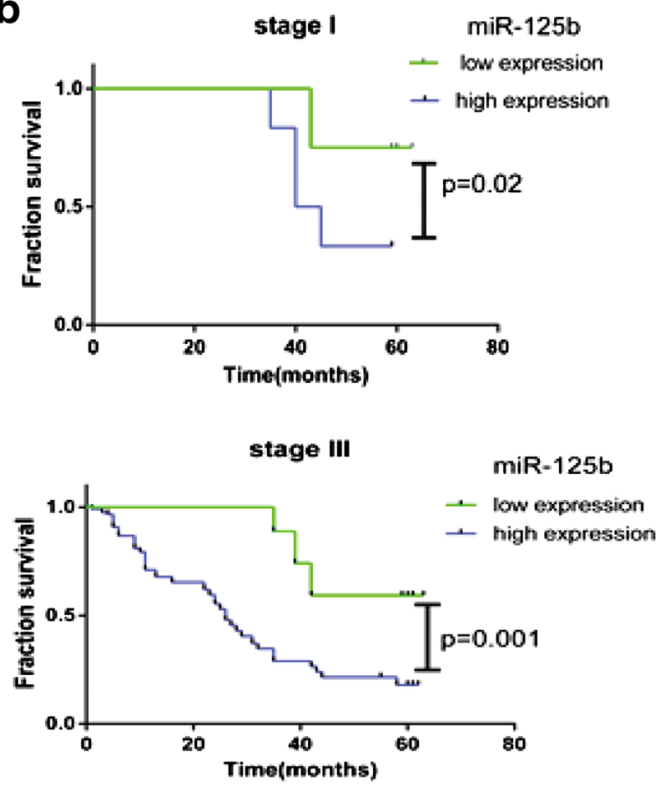

Fig. 6 a In situ hybridization (ISH) analysis of miR-125b expression in gastric cancer tissue and noncancerous human gastric mucosa. (1) $\mathrm{miR}-125 \mathrm{~b}$ was negative in noncancerous gastric mucosa (2) miR$125 \mathrm{~b}$ was highly expressed in moderately differentiated adenocarcinoma (3) miR-125b was highly expressed in poorly differentiated adenocarcinoma. b Kaplan-Meier curves with univariate analyses

$(P=0.415)$, or regional lymph nodes $(P=0.086)$. The study suggested that a high expression of MiR-125b could be an independent and significant prognostic factor in gastric cancer.

\section{Discussion}

Several studies have indicated that MiRNAs might contribute to cancer pathogenesis. MiR-125b is a small, noncoding, multipurpose RNA molecule. It is one of the first MiRNAs to be identified, and it plays a key role in several processes in many kinds of cells, such as the control of cell proliferation, differentiation, and apoptosis [1215]. The expression of MiR-125b in cancers is controversial. Some studies have shown that MiR-125b is upregulated or downregulated in different cancers under iii

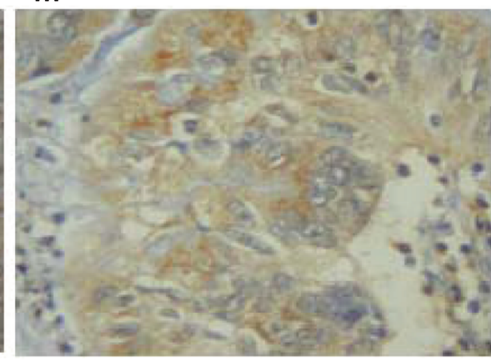

stage II

miR-125b
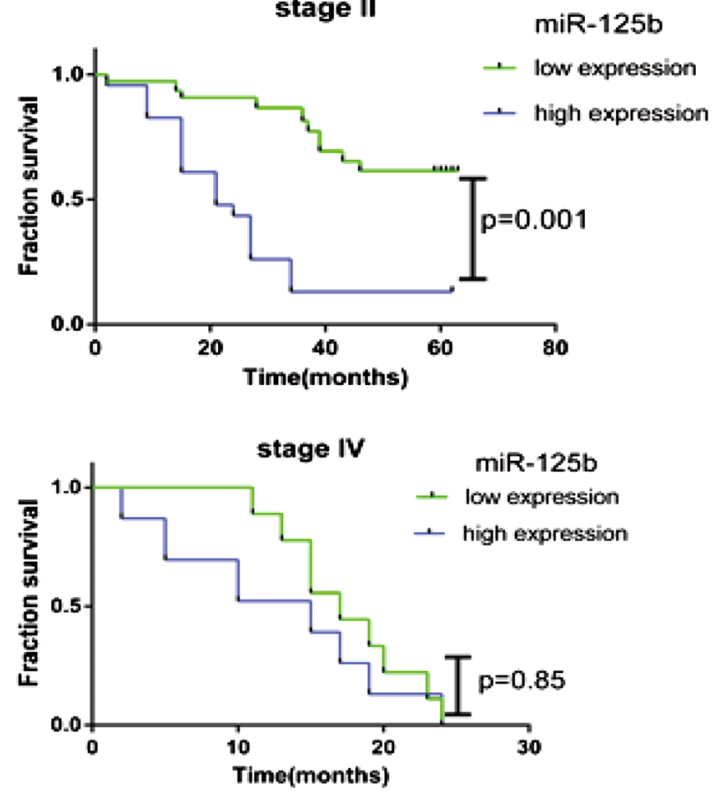

(log rank) for patients with low miR-125b expression versus high miR-125b expression. In stages I, II, and III, the 5-year survival rate of patients with high levels of miR-125b expression was significantly lower than that in patients with low levels of expression $(P<0.05)$, but in stage IV, the expression of miR-125b did not correlate with the 5-year survival rate $(P=0.24)$

pathophysiological conditions [16-19]. Although our previous study reported that MiR-125b was upregulated 4.88-fold in gastric cancer [20], the expression level and biological impact of MiR-125b in gastric cancer were never well studied. In this study, we examined the expression of MiR-125b in gastric tumor tissues and cell lines, and explored the biological role, regulation mechanism, and clinical significance in gastric cancer for the first time. As we expected, the expression of MiR-125b is upregulated in gastric cancer and in 5 gastric cancer cell lines compared to non-tumor tissues. These results suggested that MiR-125b functions as an oncogenic MiRNA in gastric cancer.

As an oncogenic MiRNA, we presumed that MiR-125b could promote gastric cancer cell proliferation, clone formation, migration, and invasion. As anticipated, cell proliferation, clone formation, migration, and invasion of 
HCG-27 and MKN-28, in which MiR-125b expression was higher in 5 gastric cancer cell lines, were significantly suppressed by transfection of MiR-125bi. In contrast, cell proliferation, clone formation, migration, and invasion were significantly promoted in MKN-45 cells with transfected MiR-125bm; therefore, we explored the tumorpromoting role for MiR-125b in gastric cancer for the first time and possible evidence of the potential usefulness of MiR-125b in MiRNA-based cancer therapy. MiR-125b has various regulating pathways in the process of cell proliferation, differentiation, and apoptosis, such as inhibiting p53-induced apoptosis during development and the stress response [14], inhibiting cell apoptosis through p53 and p38- MAPK-independent pathways in glioblastoma cells [21], targeting EPO and EPOR to correlate with metastatic potential, and expressing ERBB2/HER2 in breast cancer [22]. In this study, to explore the potential targets of MiR$125 \mathrm{~b}$ that might influence proliferation and migration ability of gastric cancer cells, PPP1CA was found among the potential targets of the MiR-125b combination predicted by TargetScan, Pictar-Vert, and Microrna. Org. Protein serine/threonine (Ser/Thr) phosphatase-1 (PP1) is one of the most highly conserved enzymes known, and it plays a central role in a range of cellular processes, including protein synthesis, RNA splicing, cell-cycle progression, and glycogen metabolism [23, 24]. A large array of regulatory subunits are associated with the PP1 catalytic subunit to determine its cellular localization and substrate specificity, mediating the control of these many physiological processes through PP1 holoenzymes [24-26]. There are 4 different isoforms of the PP1 catalytic subunit ( $a, b$, $\mathrm{c} 1$, and $\mathrm{c} 2$ ) and each can bind to many non-substrate cellular proteins that function as regulatory subunits. These subunits modify the activity of PP1 and/or target it to particular substrates. Finally, PP1 promotes the exit from mitosis and maintains cells in the G1 or G2 phase of the cell cycle. PPP1CA encodes the catalytic subunit of PP1a. The PP1a catalytic subunit can form complexes with many regulatory subunits, which regulate various cellular activities, such as the cell cycle, apoptosis, and signal transduction [25, 27]. One of the most important PP1 target proteins is $\mathrm{Rb}$, the phosphorylated product of the retinoblastoma gene that is essential in cell cycle regulation [28]. The targeting of $\mathrm{Rb}$ by PP1 contributes to the dephosphorylation and subsequent activation of $\mathrm{Rb}$, shutting down the G1/S phases of the cell cycle [28, 29]; however, the constitutive inactivation of $\mathrm{Rb}$ by maintaining highly phosphorylated $\mathrm{Rb}$ contributes to cell cycle deregulation and tumorigenesis [30-32] by sequential activation of E2F. Therefore, increased MiR-125b downregulates the expression of PPP1CA, which increases phosphorylated $\mathrm{Rb}$, in turn, by the further sequential activation of E2F and contributes to cell cycle deregulation and tumorigenesis. To verify that the putative MiR-125b binding site in the $3^{\prime} \mathrm{UTR}$ of PPP1CA was responsible for regulation by MiR-125b, we constructed vectors containing wild-type or mutant 3'UTR of PPP1CA co-transfect into MKN-28 cells with MiR-125bm, MiR-125bm NC, or none. As a result, MiR$125 \mathrm{~b}$ significantly decreased the relative luciferase activity of wild-type PPP1CA $3^{\prime} \mathrm{UTR}(\sim 60 \%)$, suggesting that MiR-125b could directly bind to the $3^{\prime}$ UTR of PPP1CA. Furthermore, we analyzed the effect of MiR-125b on the expression of PPP1CA and $\mathrm{Rb}$ protein phosphorylation. The mRNA and protein levels of PPP1CA in MiR-125bitransfected HGC-27 and MKN-28 cells were much higher than in the NC-transfected group, while the $\mathrm{Rb}$ protein phosphorylation level in MiR-125bi- transfected HGC-27 and MKN-28 cells was much lower than that in the NCtransfected group. In addition, MiR-125bi plus siPPP1CA (PPP1CA siRNA) and MiR-125bi were transfected into HGC-27 and MKN-28 cells. As a result, HGC-27 and MKN-28 co-transfected with MiR-125b inhibitor plus siPPP1CA revealed a higher effect than in those transfected with MiR-125bi on enhancing cell proliferation, clone formation, migration, and invasion ability $(P<0.01)$, suggesting that the MiR-125b promoted the proliferation, clone formation, migration, and invasion migration of gastric cancer cells by targeting PPP1CA. Taken together, our overall interpretation of these results is that the overexpression of MiR-125b leads to the downregulation of PPP1CA, which promotes $\mathrm{Rb}$ protein phosphorylation and activation of E2F. These findings indicated that MiR-125b might directly target the PPP1CA-Rb-E2F signal pathway to contribute to gastric cancer pathogenesis.

Interestingly, this high expression level of MiR-125b in gastric cancer tissues was associated with lymph node and distant metastases. In situ hybridization indicated that MiR$125 \mathrm{~b}$ was highly expressed in gastric cancer tissues and correlated with the size of the tumor, the depth of invasion, lymph nodes, distant metastasis, and TNM stage. Cox regression analysis revealed that the MiR-125b expression level was an independent prognostic factor in patients with gastric carcinoma. These results suggest that MiR-125b might play an important role in gastric tumorigenesis, progression, and prognosis.

In summary, this study provided new insights into the role of MiR-125b in human gastric cancers. It showed that MiR-125b is highly expressed in gastric tumor tissues and gastric cancer lines, and that it is able to promote gastric cancer cell proliferation, migration, and invasion in vitro by targeting downregulated PPP1CA and upregulated $\mathrm{Rb}$ phosphorylation. Furthermore, MiR-125b was an independent prognostic factor in gastric cancer. These results suggested that MiR-125b was an oncogenic MiRNA and might serve as an independent prognostic factor and therapeutic target in gastric cancer. 
Acknowledgments This work was supported by grants from the Medicine and Health Research Fund of Zhejiang Province (2012RCA005)

Conflict of interest The authors declare no conflicts of interest.

\section{References}

1. Zhan WH, Han FH. Surgical therapy of gastric cancer in china. J Pract Oncol. 2008;23:91-3.

2. Hutvágner G, Zamore PD. A microRNA in a multiple-turnover RNAi enzyme complex. Science. 2002;297:2056-60.

3. Bartels CL, Tsongalis GJ. MicroRNAs: novel biomarkers for human cancer. Clin Chem. 2009;55:623-31.

4. Nelson PT, Baldwin DA, et al. RAKE and LNA-ISH reveal microRNA expression and localization in archival human brain. RNA. 2006;12:187-91.

5. Volinia S, Calin GA, Liu CG, et al. A microRNA expression signature of human solid tumors defines cancer gene targets. Proc Natl Acad Sci. 2006;103:2257-61.

6. Iorio MV, Visone R, et al. MicroRNA signatures in human ovarian cancer. Cancer Res. 2007;67:8699-707.

7. Nam EJ, Yoon H, et al. MicroRNA expression profiles in serous ovarian carcinoma. Clin Cancer Res. 2008;14:2690-5.

8. Bloomston M, Frankel WL, et al. MicroRNA expression patterns to differentiate pancreatic adenocarcinoma from normal pancreas and chronic pancreatitis. JAMA. 2007;297:1901-8.

9. Shi XB, Xue L, et al. An androgen-regulated miRNA suppresses Bak1 expression and induces androgen-independent growth of prostate cancer cells. Proc Natl Acad Sci. 2007;2007(104): 19983-8.

10. Bousquet M, Quelen C, Rosati R, et al. Myeloid cell differentiation arrest by miR-125b-1 in myelodysplastic syndrome and acute myeloid leukemia with the $\mathrm{t}(2 ; 11)(\mathrm{p} 21 ; \mathrm{q} 23)$ trans-location. J Exp Med. 2008;205:2499-506.

11. Song LB, Liao WT, Mai HQ, et al. The clinical significance of twist expression in nasopharyngeal carcinoma. Cancer Lett. 2006;242:258-65.

12. Zhao ZS, Wang YY, Chu YQ, et al. SPARC is associated with gastric cancer progression and poor survival of patients. Clin Cancer Res. 2010;16:260-8.

13. Le MT, Xie H, Zhou B, et al. MicroRNA-125b promotes neuronal differentiation in human cells by repressing multiple targets. Mol Cell Biol. 2009;29:5290-305.

14. Le MT, Teh C, Shyh-Chang N, et al. MicroRNA-125b is a novel negative regulator of p53. Genes Dev. 2009;23:862-76.

15. Lee YS, Kim HK, Chung SM, et al. Depletion of human microRNA miR-125b reveals that it is critical for the proliferation of differentiated cells but not for the down-regulation of putative targets during differentiation. J Biol Chem. 2005;280:16635-41.
16. Vriens MR, Weng J, Suh I, et al. MicroRNA expression profiling is a potential diagnostic tool for thyroid cancer. Cancer. 2012;118:3426-32.

17. Pallante P, Visone R, Ferracin M, et al. MicroRNA deregulation in human thyroid papillary carcinomas. Endocr Relat Cancer. 2006;13:497-508.

18. Jia HY, Wang YX, Yan WT, et al. MicroRNA-125b functions as a tumor suppressor in hepatocellular carcinoma cells. Int J Mol Sci. 2012;13:8762-74.

19. Henson BJ, Bhattacharjee S, O'Dee DM, et al. Decreased expression of miR-125b and miR-100 in oral cancer cells contributes to malignancy. Genes Chromosomes Cancer. 2009;48:569-82.

20. Wang YY, Ye ZY, Zhao ZS, et al. Clinicopathologic significance of miR-10b expression in gastric carcinoma. Human Pathology. 2013;44:1278-85.

21. Wu N, Lin X, Zhao X, et al. MiR-125b acts as an oncogene in glioblastoma cells and inhibits cell apoptosis through p53 and p38MAPK-independent pathways. $\mathrm{Br} \mathrm{J}$ Cancer. 2013;109(11):2853-63.

22. Ferracin M, Bassi C, Pedriali M. miR-125b targets erythropoietin and its receptor and their expression correlates with metastatic potential and ERBB2/HER2 expression. Mol Cancer. 2013;12(1): 130 .

23. Aggen JB, Nairn AC, Chamberlin R. Regulation of protein phosphatase-1. Chem Biol. 2000;7:R13-23.

24. Ceulemans H, Bollen M. Functional diversity of protein phosphatase-1, a cellular economizer and reset button. Physiol Rev. 2004;84:1-39.

25. Cohen PT. Protein phosphatase 1-targeted in many directions. J Cell Sci. 2002;115:241-56.

26. Hendrickx A, Beullens M, Ceulemans H, Den Abt T, Van Eynde A, et al. Docking motif-guided mapping of the interactome of protein phosphatase-1. Chem Biol. 2009;16:365-71.

27. Ceulemans H, Bollen M. Functional diversity of protein phosphatase-1, a cellular economizer and reset button. Physiol Rev. 2004;84:1-39.

28. Berndt N. Protein dephosphorylation and the intracellular control of the cell number. Front Biosci. 1999;4:22-42.

29. Liu CW, Wang RH, Dohadwala M, et al. Inhibitory phosphorylation of PP1alpha catalytic subunit during the $\mathrm{G}(1) / \mathrm{S}$ transition. J Biol Chem. 1999;274:29470-5.

30. Malumbres M, Barbacid M. Cell cycle, CDKs and cancer: a changing paradigm. Nat Rev Cancer. 2009;9:153-66.

31. Sherr CJ. Principles of tumor suppression. Cell. 2004;116: 235-46.

32. Noonan EJ, Place RF, Basak S, et al. miR-449a causes Rbdependent cell cycle arrest and senescence in prostate cancer cells. Oncotarget. 2010;1:349-58. 